\title{
Evaluation and comparative analysis of minimal formalin containing compound fixatives
}

\author{
S. Muthuselvi ${ }^{1}$,J. Suresh Durai ${ }^{2, *}$, Johnsy Merla ${ }^{3}$, K. Shantaraman ${ }^{4}$ \\ ${ }^{\mathbf{1}}$ Assistant Professor, Dept. of Pathology, Thoothukudi Medical College, Tamil Nadu, ${ }^{2}$ Professor, ${ }^{\mathbf{3}}$ Assistant Professor, ${ }^{4}$ Professor \\ \& HOD, Dept. of Pathology, Tirunelveli Medical College, Tamil Nadu, India
}

*Corresponding Author:
Email: srshdr@yahoo.com

Received: $13^{\text {th }}$ December, 2017

Accepted: $23^{\text {rd }}$ February, 2018

\begin{abstract}
Introduction: Fixation is the crucial step in the practice of diagnostic pathology. Formalin has been used as a general fixative for more than a century. The IARC (International Agency for Research on Cancer) reported that there is a causal association between formalin exposure and leukemia and nasopharyngeal carcinoma. Hence this study was done to find an alternative fixative to minimize formalin exposure in histopathology laboratory.

Materials and Methods: 100 specimens were fixed in 3 different minimal formalin containing compound fixatives composed of ethanol, glycerin, formalin (EFG fixatives)and hypotonic saline at fixation time of 10, 8 and 7 hours. The processed specimens were analyzed for fixation artifacts, staining characteristics and cytoarchitectural features and compared with conventional formalin fixation.

Results: Cytoarchitectural features were well preserved as conventional formalin fixation in both EFG I and EFG II at 8 and 10 hours of fixation, and with inadequate fixation characters at 7 hours. EFG II was found to evaporate less formaldehyde vapor than $10 \%$ buffered formalin and fixation time reduced upto 8 hours. EFG III resulted in poor preservation of tissues in all three fixation times.

Conclusion: As formaldehyde is a group 1 human carcinogen, it should be replaced by less toxic fixatives in histopathology laboratories. The present study highlights that minimal formalin containing EFG fixatives can be easily prepared in the laboratory and their effectiveness in routine histopathology examination is comparable with conventional formalin fixation.
\end{abstract}

Keywords: Compound fixatives, Architecture, Fixation, Formalin toxicity, Histopathology.

\section{Introduction}

Fixation is an important step in diagnostic pathology as it maintains tissues in life like manner and formalin is used as routine fixative in histopathology laboratory. The main motives for the popularity of formalin are its low cost, allows special histological stains, preserves morphological features and long term storage of tissues. The laboratory preparation of formalin is a straightforward procedure. However, the toxicity of formalin is emerging as the main reason to abolish it as the commonest fixative used in laboratories. Initial report from the IARC (International Agency for Research on Cancer) link formaldehyde exposure and leukemia. The national and international level agencies that monitor formaldehyde exposure set stringent limits for formaldehyde exposure which ranges from $0.016 \mathrm{ppm}$ TWA (time weighted average) to $2 \mathrm{ppm}$ for STEL (short term exposure limit). ${ }^{3}$ An attempt has been made in this study to minimize formalin exposure by reducing the formalin concentration in a new compound fixative. These compound fixatives are evaluated for their efficacy and bio safety.

\section{Materials and Methods}

A group of three minimal formalin containing fixatives (EFG fixatives) were prepared with varying concentrations of ethanol, glycerin, formalin.
Compound fixative 1 was prepared with $10 \%$ Formalin $7 \mathrm{ml}$, Ethanol $20 \mathrm{ml}$, Glycerin 5ml, Methylene Blue $0.05 \mathrm{ml}$. The second fixative was prepared with $10 \%$ Formalin $6 \mathrm{ml}$, Ethanol $30 \mathrm{ml}$, Glycerin 5ml, Methylene blue $0.05 \mathrm{ml}$, The third composition was prepared with $10 \%$ Formalin $5 \mathrm{ml}$, Ethanol $40 \mathrm{ml}$, Glycerin $5 \mathrm{ml}$, Methylene blue $0.05 \mathrm{ml}$. Buffer - $4 \mathrm{~g}$ of Sodium dihydrogen phosphate monohydrate, $6 \mathrm{~g}$ of Anhydrous disodium hydrogen phosphate was added to all the three compound fixatives.

The $\mathrm{pH}$ of the fixatives was maintained between 7.2 to 7.4 using buffer. And the fixatives were reconstituted with $0.7 \%$ hypotonic saline to $100 \mathrm{ml}$. Fixatives were named EFG I, II, III for their major constituents Ethyl alcohol, Glycerin and Formalin. Ethanol as a dehydrant fixative, it will produce cell shrinkage. To overcome this, hypotonic saline was added. Glycerin was added to minimize evaporation. Methylene blue was added to monitor the color of fixatives and subsequent dehydrants and to avoid the tendency to smell the solutions. The prepared solutions were light blue in color.

Multiple human tissue materials (100 specimens) of varying sites and lesions were utilized for this study. Among them 35 specimens were fixed in fixative 1 (EFG I), 35 were fixed in fixative 2 (EFG II) and 30 specimens were fixed in fixative 3(EFG III). Fixation time was titrated between 7 to 10 hours. Conventional 
tissue processing was completed in 9 Hours. Processed tissues were embedded in paraffin wax. Then the sections were taken 4 micron thickness by using microtome and stained with routine hematoxylin and eosin staining. Fixation artifacts, staining characteristics, architecture, nuclear and cytoplasmic details were analyzed by two independent pathologists. Scoring was given based on the scoring system which was used in our pilot study. ${ }^{1}$ Combined nuclear, cytoplasmic and architectural features were scored between 0- 9. A combined total score of 9 was given to nuclear, cytoplasmic and architectural features of all the tissues well fixed in 24 hours conventional $10 \%$ NBF (Neutral Buffered Formalin) which is considered as absolute fixation.

Nuclear features were assessed based on following features - nuclear preservation, nucleolar preservation, nuclear size, regularity of the nuclear membrane, mitotic figures and chromatin pattern whether fine, coarse, granular/ reticular pattern. The score was given between 0-3. Cytoplasmic features were assessed by colour of cytoplasm, abundance, cytoplasmic granules and mucin differentiation and the score was given between 0-3. Architectural features were assessed based on distortion, shrinkage artifacts, cracking and formalin pigments and score was given between 0-3 .

The fixation time and amount of reagents used in each fixation were evaluated and compared with conventional fixation procedures. The results were tabulated and analyzed with Mann-Whitney U test. P value $<0.05$ is considered as statistically significant.

The concentration of formaldehyde vapor in our compound fixatives was compared with conventional $10 \%$ NBF by using schiff's reagent. No.1 whatman filter paper was soaked in schiff's reagent and dried in air. Two glass beakers of $9 \mathrm{~cm}$ in length and $7.5 \mathrm{~cm}$ in diameter were taken and labeled as beaker $\mathrm{A}$ and beaker B. $10 \mathrm{ml}$ of $10 \% \mathrm{NBF}$ was poured into beaker A and $10 \mathrm{ml}$ of fixative 2 was poured into beaker B. Both beakers were closed by whatman paper (schiff's reagent soaked) and allowed to stand. The time taken for the filter papers to change colour into pink/magenta was noted.

\section{Results}

In our previous study by Suresh durai et al, the cytoarchitectural features of EFG I fixative were compared with conventional formalin. Nuclear, cytoplasmic and architectural features of tissues fixed in EFG I was compared with conventional formalin fixed tissues (table 1) and the effectiveness of this compound fixatives was comparable to conventional formalin fixation with an improved air quality of the working laboratory and considerably reduced formalin vapor density.

Table 1: Fixation time and cytoarchitectural details in EFG I

\begin{tabular}{|c|c|c|c|c|c|c|}
\hline \multirow{2}{*}{\multicolumn{2}{|c|}{ Nuclear features }} & \multicolumn{4}{|c|}{ Score } & \multirow{3}{*}{$\frac{\text { P value }}{\mathrm{n} / \mathrm{a}}$} \\
\hline & & 3 & 2 & 1 & $\mathbf{0}$ & \\
\hline \multirow{4}{*}{ 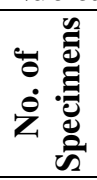 } & $10 \% \mathrm{NBF}$ & 35 & nil & nil & nil & \\
\hline & EFG I,7 Hrs & 15 & 20 & nil & nil & $<0.0001$ \\
\hline & EFG I, 8Hrs & 34 & 1 & nil & nil & 0.325 \\
\hline & EFG I,10 Hrs & 35 & nil & nil & nil & $\mathrm{n} / \mathrm{a}$ \\
\hline \multirow{2}{*}{\multicolumn{2}{|c|}{ Cytoplasmic features }} & \multicolumn{4}{|c|}{ Score } & \\
\hline & & 3 & 2 & 1 & $\mathbf{0}$ & P value* \\
\hline \multirow{4}{*}{ 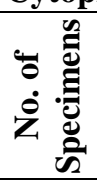 } & $10 \% \mathrm{NBF}$ & 35 & nil & nil & nil & $\mathrm{n} / \mathrm{a}$ \\
\hline & EFG I, 7Hrs & 10 & 25 & nil & nil & $<0.0001$ \\
\hline & EFG I, 8Hrs & 32 & 3 & nil & nil & 0.083 \\
\hline & EFG I, $10 \mathrm{Hrs}$ & 32 & 3 & nil & nil & 0.083 \\
\hline \multirow{2}{*}{\multicolumn{2}{|c|}{ Architectural features }} & \multicolumn{4}{|c|}{ Score } & \\
\hline & & 3 & 2 & 1 & $\mathbf{0}$ & P value* \\
\hline \multirow{4}{*}{ 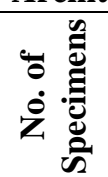 } & $10 \% \mathrm{NBF}$ & 35 & nil & nil & nil & $\mathrm{n} / \mathrm{a}$ \\
\hline & EFG I, 7 Hrs & 12 & 23 & nil & nil & $<0.0001$ \\
\hline & EFG I, 8Hrs & 33 & 2 & nil & nil & 0.160 \\
\hline & EFG I, $10 \mathrm{Hrs}$ & 33 & 2 & nil & nil & 0.160 \\
\hline
\end{tabular}

*Mann Whitney U test

There is no significant difference between $10 \%$ NBF and EFG I at 10 and 8 hours fixation. Hence EFG I is found to be qualitatively comparable to $10 \% \mathrm{NBF}$ in preserving nuclear, cytoplasmic and architectural features at 10 and 8 hours fixation. At 7 hours more than half of the tissues received suboptimal score.
In this study, the cytoarchitectural features of EFG II and EFG III fixative were compared with conventional formalin. Tissues fixed in our newer compound fixatives were light blue in colour. Texture of tissues after fixation was same as tissues fixed in conventional $10 \%$ NBF. There was no difficulty in cutting and sectioning. 
Table 2: Fixation time and cytoarchitectural details in EFG II

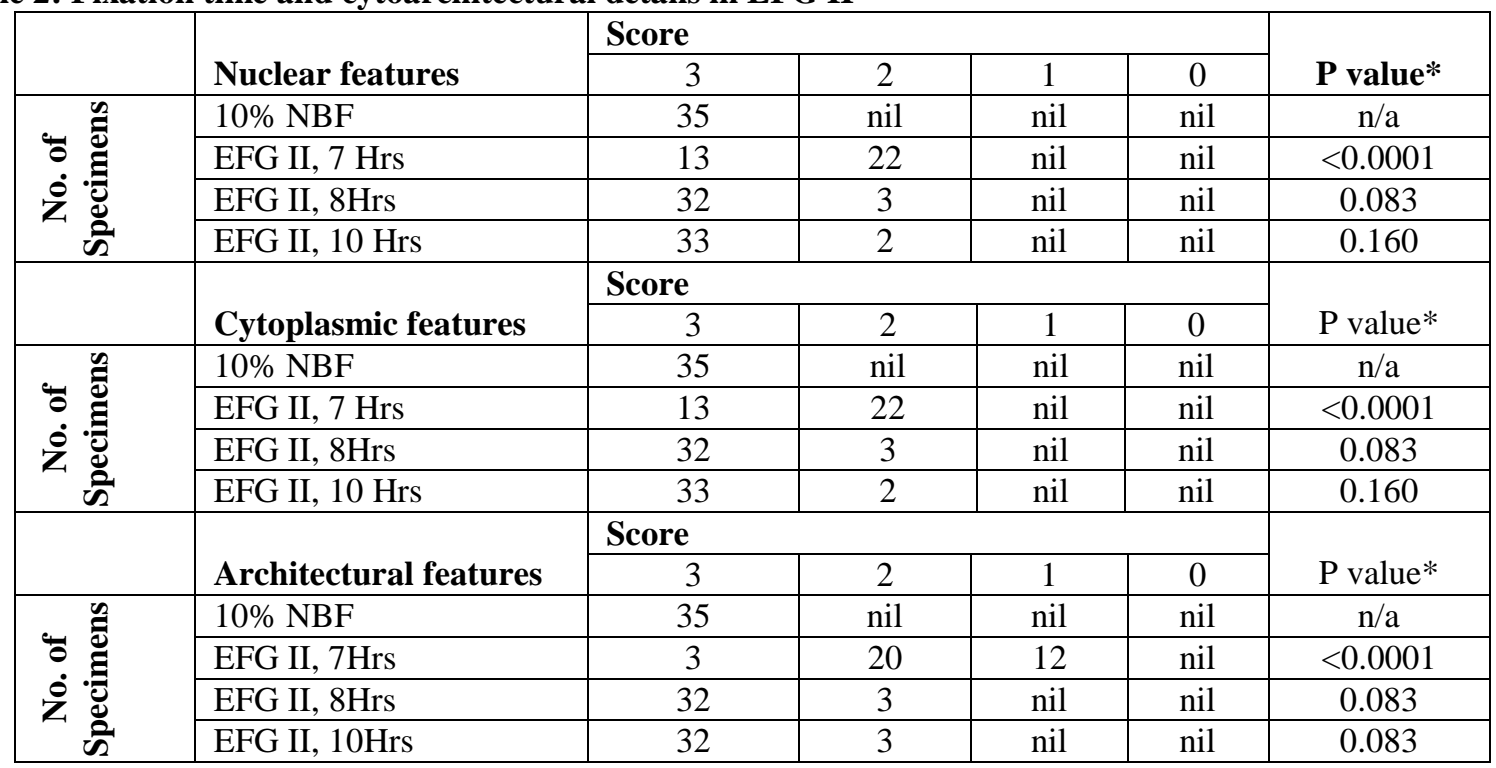

*Mann Whitney U test

Regarding nuclear details, there is no significant difference between tissues fixed in 10 and 8 hours. So EFG II also gives optimal fixation at 10 and 8 hours. There is significant difference between 10 and 7 hours fixation. This indicates 7 hours fixation results in suboptimal fixation.

On comparing cytoplasmic details between 10 and 8 hours more than 30 cases fixed in EFG II received score 3. At 7 hours fixation more than half of the cases (27) got score 2 because of cytoplasmic shrinkage. There is no significant difference between 10 and 8 hours fixation. Between 10 and 7 hours, there is significant difference. This indicates optimal fixation achieved at 8 hours fixation in EFG II.

Architecture features of tissues fixed in EFG II were compared between 10 and 8 hours and between 10 and 7 hours. Both 10 and 8 hours fixation, 32 specimens got score 3 . At 7 hours, 20 cases got score 2 because they showed shrinkage artifact, distortion and cracking. The above table (table 2) shows fixative 2 scored equal number of maximum score at 10 and 8 hours. So $\mathrm{p}$ value is not available, EFG II produces similar results in both fixation hours. There is significant difference between 10 and 7 hours. Tissues fixed in 10 hours fixation gives better architecture details than 7 hours fixation.

Table 3: Fixation time and cytoarchitectural details in EFG III

\begin{tabular}{|c|c|c|c|c|c|c|}
\hline \multirow{2}{*}{\multicolumn{2}{|c|}{ Nuclear features }} & \multicolumn{4}{|l|}{ Score } & \multirow[b]{2}{*}{ P value* } \\
\hline & & 3 & 2 & 1 & 0 & \\
\hline \multirow{4}{*}{ 它 } & $10 \% \mathrm{NBF}$ & 30 & nil & nil & nil & $\mathrm{n} / \mathrm{a}$ \\
\hline & EFG III, 7 Hrs & 2 & 22 & 6 & nil & $<0.0001$ \\
\hline & EFG III, 8Hrs & 6 & 23 & 1 & nil & $<0.0001$ \\
\hline & EFG III, $10 \mathrm{Hrs}$ & 6 & 22 & 2 & nil & $<0.0001$ \\
\hline \multirow{2}{*}{\multicolumn{2}{|c|}{ Cytoplasmic features }} & \multicolumn{4}{|l|}{ Score } & \multirow[b]{2}{*}{$P$ value* } \\
\hline & & 3 & 2 & 1 & 0 & \\
\hline \multirow{4}{*}{ 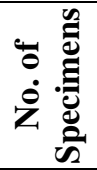 } & $10 \% \mathrm{NBF}$ & 30 & nil & nil & nil & $\mathrm{n} / \mathrm{a}$ \\
\hline & EFG III, 7 Hrs & 5 & 18 & 7 & nil & $<0.0001$ \\
\hline & EFG III, 8Hrs & 5 & 21 & 4 & nil & $<0.0001$ \\
\hline & EFG III, $10 \mathrm{Hrs}$ & 5 & 18 & 7 & nil & $<0.0001$ \\
\hline \multirow{2}{*}{\multicolumn{2}{|c|}{ Architectural features }} & \multicolumn{4}{|l|}{ Score } & \multirow[b]{2}{*}{$P$ value* } \\
\hline & & 3 & 2 & 1 & 0 & \\
\hline \multirow{4}{*}{ 苛 } & $10 \% \mathrm{NBF}$ & 30 & nil & nil & nil & $\mathrm{n} / \mathrm{a}$ \\
\hline & EFG III, 7 Hrs & nil & 10 & 20 & nil & $<0.0001$ \\
\hline & EFG III, 8Hrs & nil & 19 & 11 & nil & $<0.0001$ \\
\hline & EFG III, $10 \mathrm{Hrs}$ & nil & 17 & 13 & nil & $<0.0001$ \\
\hline
\end{tabular}

*Mann whitney U test 
Marked nuclear shrinkage, less defined chromatin pattern, less prominent nuclear membrane and nucleolus were observed in all the three fixation times (table 3). Cytoplasmic features between EFG III and conventional formalin were compared. In all three fixation hours, more than half of the tissues got score 2 because of cytoplasmic shrinkage. 4 to 7 cases showed even more shrinkage and received score 1 . In all three fixation hours architectural features received score 2 and 1 because of more shrinkage artifacts, distortion and cracking.

As there was significant difference in nuclear, cytoplasmic and architectural features in all three fixation times (7, 8 and 10 hours) of EFG III compared to conventional formalin, only the cytoarchitectural features of the other two fixatives (EFG I and EFG II) were compared for their superiority.

Table 4: Comparison of EFG I and EFG II (Nuclear features)

\begin{tabular}{|c|c|c|c|c|c|c|c|}
\hline \multirow[t]{2}{*}{ 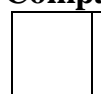 } & \multirow[b]{2}{*}{ Time } & \multirow[b]{2}{*}{ Fixatives } & \multicolumn{4}{|c|}{ Score } & \multirow[b]{2}{*}{ P value* } \\
\hline & & & 3 & 2 & 1 & 0 & \\
\hline \multirow{6}{*}{ 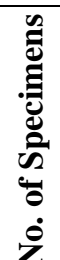 } & \multirow[b]{2}{*}{7 hours } & EFG I & 15 & 20 & nil & nil & \multirow[t]{2}{*}{0.962} \\
\hline & & EFG II & 13 & 22 & nil & nil & \\
\hline & \multirow[b]{2}{*}{8 hours } & EFG I & 34 & 1 & nil & nil & \multirow[t]{2}{*}{0.505} \\
\hline & & EFG II & 32 & 3 & nil & nil & \\
\hline & \multirow[b]{2}{*}{10 hours } & EFG I & 35 & nil & nil & nil & \multirow[t]{2}{*}{0.513} \\
\hline & & EFG II & 33 & 2 & nil & nil & \\
\hline
\end{tabular}

*Mann Whitney U test

At 7 hours, both fixatives (EFG I and EFG II) received score 2 because they showed less prominent above nuclear features. Almost all cases fixed in both EFG I and EFG II at 10 and 8 hours fixation received a full score of 3 as they preserved well defined chromatin pattern, distinct nuclear membrane, nucleoli and mitotic figures (Table 4).

Table 5: Comparison of EFG I and EFG II (cytoplasmic features):

\begin{tabular}{|c|c|c|c|c|c|c|c|}
\hline & \multirow[b]{2}{*}{ Time } & \multirow[t]{2}{*}{ Fixatives } & \multicolumn{4}{|l|}{ Score } & \multirow[b]{2}{*}{ P value } \\
\hline & & & 3 & 2 & 1 & 0 & \\
\hline \multirow{6}{*}{ 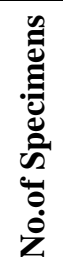 } & \multirow[b]{2}{*}{7 hours } & EFG I & 10 & 25 & nil & nil & \multirow[t]{2}{*}{0.003} \\
\hline & & EFG II & 13 & 22 & nil & nil & \\
\hline & \multirow[b]{2}{*}{8 hours } & EFG I & 32 & 3 & nil & nil & \multirow[t]{2}{*}{$\mathrm{n} / \mathrm{a}$} \\
\hline & & EFG II & 32 & 3 & nil & nil & \\
\hline & \multirow[b]{2}{*}{10 hours } & EFG I & 32 & 3 & nil & nil & \multirow[b]{2}{*}{1.000} \\
\hline & & EFG II & 33 & 2 & nil & nil & \\
\hline
\end{tabular}

*Mann Whitney U test

Cytoplasmic features were compared among EFG I and II. At 7 hours fixation, more than half of the specimens fixed in both fixatives got score 2 because of shrinkage artifact and cracking especially thyroid and nodal tissues. At 8,10 hours fixation, more than 30 cases fixed in EFG II received score 3 (Table 5).

Table 6: Comparison of EFG I and EFG II (Architectural features)

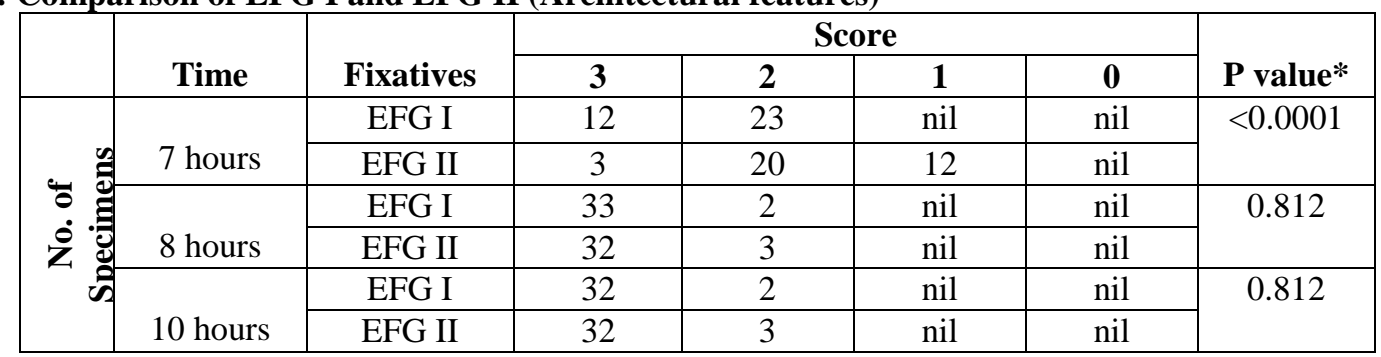

*Mann Whitney U test

There is no significant difference between EFG I and II at both 8 and 10 hours fixation (Table 6). So both fixatives equally preserve architectural detail at 8 and 10 hours.
Formaldehyde vapor from the compound fixatives were qualitatively measured and compared with conventional $10 \% \mathrm{NBF}$ by Schiff test. In this test, filter paper over beaker A $(10 \% \mathrm{NBF})$ started to change colour in 10 minutes and completely changed colour in 
25 minutes. Whereas filter paper over beaker B (fixative 2) started to change colour in 55 minutes and completely changed in 90 minutes.

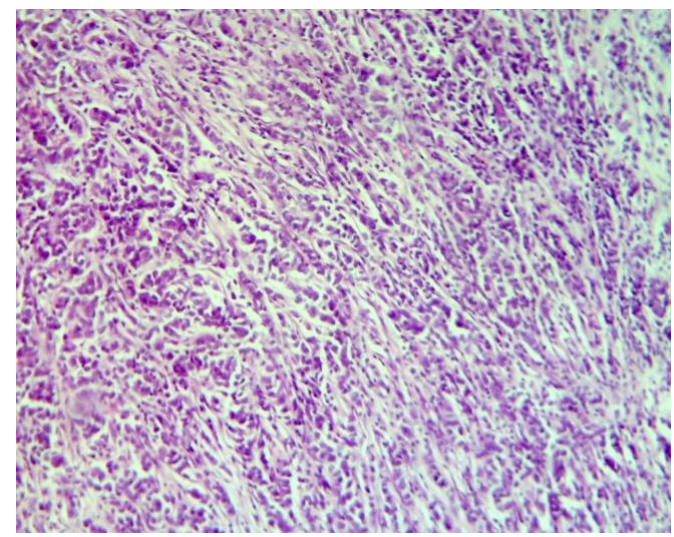

Fig. 1: Photomicrograph showing histopathological features of Invasive ductal carcinoma breast EFG I fixative, 8 hours. H\& E (10x)

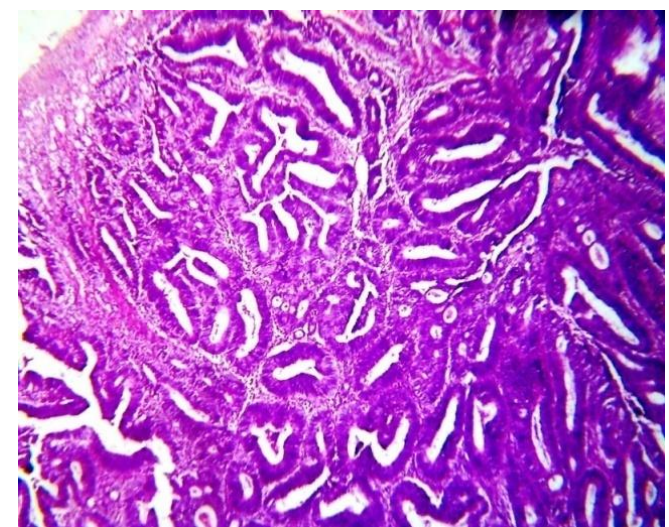

Fig. 2: Photomicrograph showing histopathological features of well differentiated adenocarcinoma colon EFG II fixative, 10 hours. H\& E (4x)

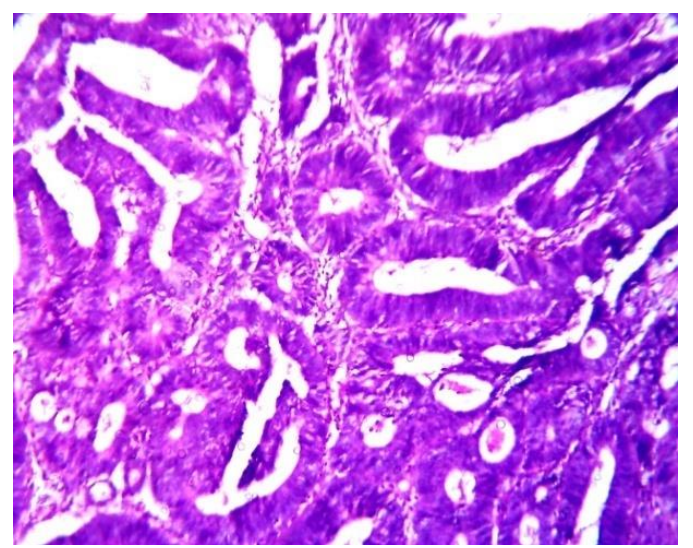

Fig. 3: Photomicrograph showing histopathological features of well differentiated adenocarcinoma colon, EFG II fixative, 10 hours. H\& E (10x)

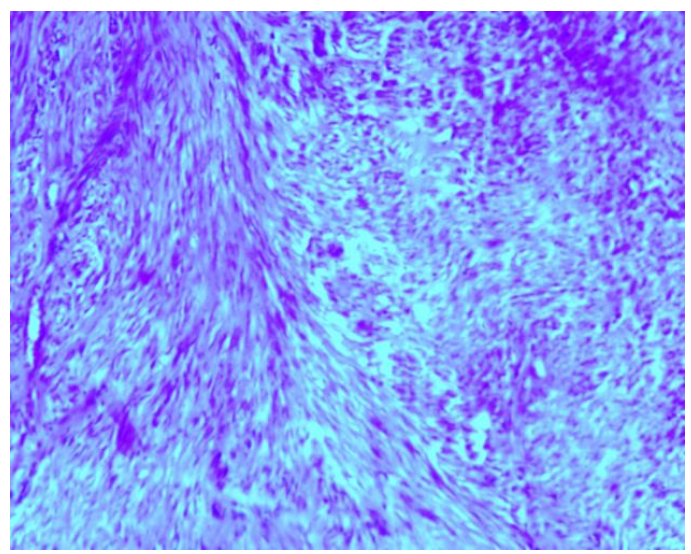

Fig. 4: Photomicrograph showing histopathological features of leiomyoma, EFG II fixative, 8 hours H\&E(10x)

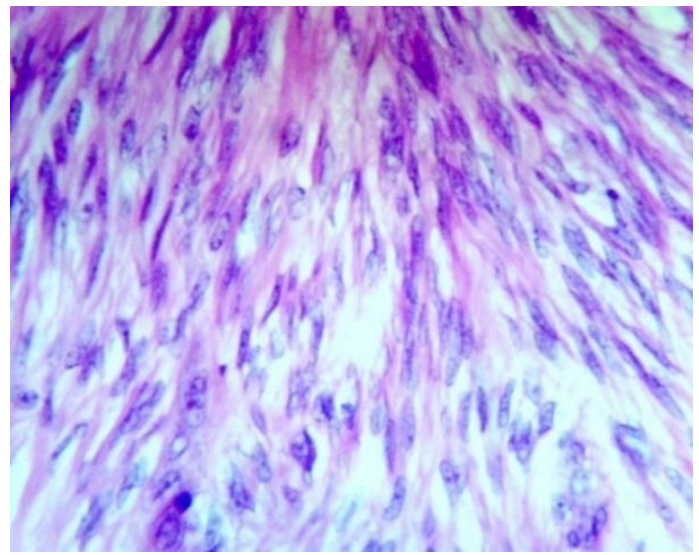

Fig. 5: Photomicrograph showing histopathological features of Leiomyoma, EFG II fixative, 8 hours H\&E(40x) 

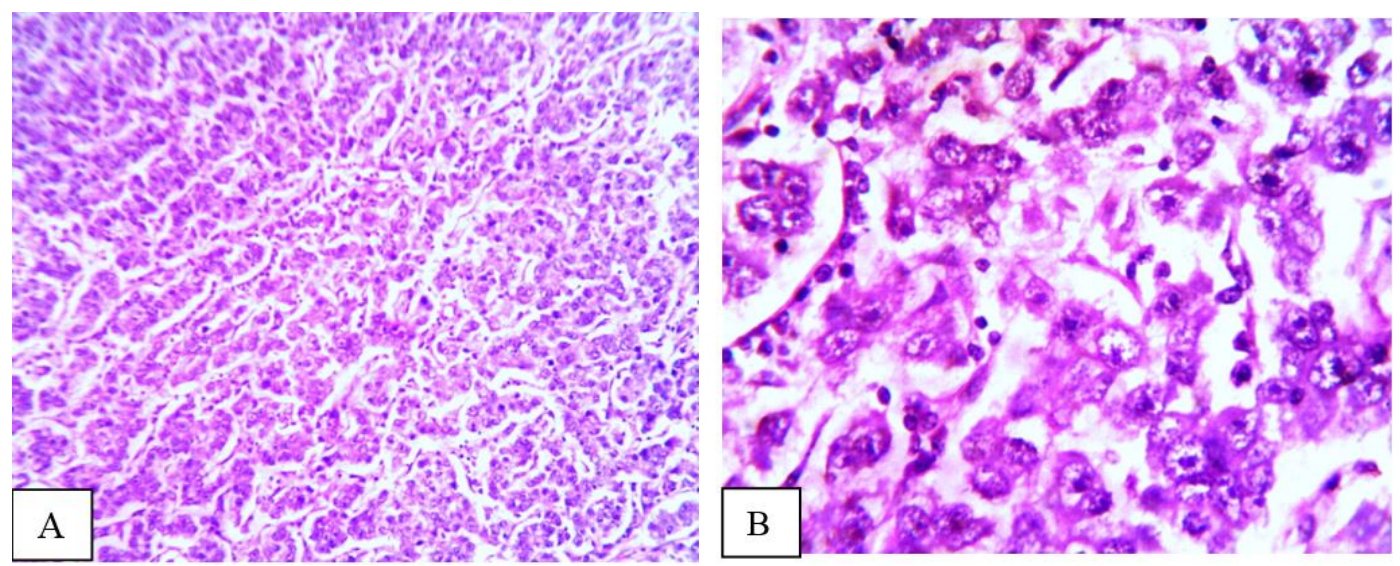

Fig. 6A: Photomicrograph showing histopathological features of dysgerminoma ovary, EFG II fixative, 8 hours H\&E(10x); B: Photomicrograph showing histopathological features of dysgerminoma ovary, EFG II fixative, 8 hours $H \& E(40 x)$.

\section{Discussion}

In the study by Suresh Durai et al, conducted in our centre earlier, formalin concentration was reduced from 10 to $7 \%$ (EGF I). This study showed that the cytoarchitectural features were comparable with that of tissues fixed in $\mathrm{NBF}^{1}$. In the present study we have tried to minimize formalin exposure in histopathology laboratory by reducing formalin concentration. A total of 100 specimens in three different compositions of EFG fixatives were used. In EFG I, formalin concentration was reduced from 10 to $7 \%$. Alcohol concentration was $20 \%$. To minimize the evaporation of absolute ethanol, we have added glycerol. Ethanol is a dehydrant coagulative fixative, it removes water molecules from tissues leads to shrinkage of cells. To overcome this, $0.7 \%$ hypotonic saline was added. Methylene blue was added to monitor spillage and contamination of subsequent dehydrants in processing. The $\mathrm{pH}$ of the solution was maintained under 7 to 7.2 by adding sodium dihydrogen phosphate monohydrate and anhydrous disodium hydrogen phosphate. In fixative 2 (EFG II) formalin concentration was further reduced to $6 \%$. Ethanol concentration was increased from 20 to $30 \%$. Fixative 3 (EFG III), ethanol concentration was further increased to $40 \%$. Formalin concentration was reduced to $5 \%$. Glycerin and methylene blue concentration was similar in all the three fixatives. The fixation characteristics and cytomorphological features of fixatives were analyzed.

The study by Cathy. B. Moelans et al found that tissues fixed in Finefix and RCL2 were to be paler when compared to specimens fixed with NBF. ${ }^{5}$ The study by Cristina Zanini et al showed that tissues fixed in PAGA, ZBF, Z7, RCL2 and Cell Block (alternative fixatives) do not change the color in in a similar manner as formalin. ${ }^{3}$ Tissues fixed in our fixatives were light blue in colour and it does not interfere with macroscopic analysis. Another factor is that the odour associated with compound fixatives is less irritant than formalin. Regarding texture of tissues,
Cathy.B.Moelans et.al reported that tissues fixed using F-solv and Finefix were found to be more rigid, and tissue fixed in RCL2 was much softer and comparatively more slippery. ${ }^{5}$ Cristina Zanini et al found that tissues fixed in alternative fixatives were suitable for microtomy. ${ }^{3}$ Cathy.B.Moelans et al reported that tissue fixed using RCL2 were softer and slippery, making cutting difficult. ${ }^{5}$ Textures of the tissues fixed by compound fixatives are suitable for microtomy.

Rate of fixation time depends on the rate at which diffusion of fixative into the tissue occurs and the rate at which chemical reactions with various components occurs. The study by Cathy.B.Moleans et al, penetration speed of alcohol based fixatives was found to be faster than $10 \% \mathrm{NBF}^{5}$.In the present study, fixative 1 shows there is no significant difference between tissues fixed at 10 and 8 hours fixation in all three morphological features (nuclear, cytoplasm and architectural details). But there is significant difference between tissues fixed at 10 and 7 hours fixation in all three parameters. Fixative 2 also shows there is no significant difference between tissues fixed at 10 and 8 hours fixation. But there is significant difference between tissues fixed at 10 and 7 hours fixation in all three parameters. This indicates 7 hours fixation is inadequate. 8 hours fixation in both EFG I and II is optimum for histopathological evaluation.

In the study by Cristina Zanini et.al, nuclear features were better preserved in alcohol based fixatives. ${ }^{3}$ L. Benerini Gatta et al - Bouin fixative showed higher resolution in the nucleus ${ }^{4}$. In the study by Cathy.B.Moelans et al demonstrated highest score for nuclear and cytoarchitectural features tissues fixed in NBF and lowest for FineFIX ${ }^{5}$. On comparing nuclear features of EFG I and 10\% NBF, there is no significant difference between them at 10 and 8 hours fixation. Tissues fixed with EFG II also compared with conventional formalin fixed tissues. And there is no significant difference between them at 10 and 8 hours. So EFG II is equally comparable with conventional 
formalin in nuclear details preservation. On comparison between EFG I and II, there is also no significant difference. So both fixatives are equally comparable with conventional formalin fixation. Nucleolus, chromatin and mitotic figures are better demonstrated in these compound fixatives. The study by L. Benerini Gatta et al found that there was no differences in cytoplasmic and nuclear morphology between alternative fixatives and formalin ${ }^{6}$. In the study by Mahdiieh Ghoddosi et al, RCL2 fixed tissues got slightly better score for cytoplasmic features ? Regarding cytoplasmic features, the present study demonstrates that there is no significant difference between EFG I and 10\% NBF. And also there is no significant difference between EFG II and 10\% NBF. Cytoplasmic features also compared between EFG I and II that showed no significant difference. So both fixatives are comparable with conventional formalin in cytoplasmic features preservation. There is significant difference between EFG III and 10\% NBF.

In a study by Cristina Zainini et al, alcohol based fixatives showed shrinkage artifacts especially when concentration of alcohol is more than $50 \%$. Fixatives containing zinc also had shrinkage artifacts ${ }^{3}$. In the study by Mahdiieh Ghoddosi et al, more than $90 \%$ cases in their study received good score for morphological features ${ }^{2}$. On comparing architectural features, it was observed that there is no significant difference between EFG I and conventional formalin at 10 and 8 hours fixation. EFG II also shows that there is no significant difference at 10 and 8 hours fixation when compared with conventional formalin. But there is significant decline in the quality of fixation by EFG III when formalin concentration is reduced to $5 \%$. From these findings we have found that both EFG I and II results in good morphological features comparable with conventional 24 hours formalin fixation. EFG II is better than I because it gives good results with minimal formalin concentration (6\%). EFG II is found to evaporate less formaldehyde vapor than $10 \% \mathrm{NBF}$ and fixation time is reduced up to 8 hours.

\section{Conclusion}

This study demonstrates that the minimal formalin containing fixative EFG II can be easily prepared in the laboratory with inexpensive easily available laboratory chemicals. It significantly improves the air quality of surgical pathology laboratory by effectively reducing the formalin vapor. EFG II fixative is found to be a suitable fixative for routine surgical specimens. Reduced fixation time, reduced formalin vapor and preservation of excellent cytoarchitectural features in $\mathrm{H}$ $\&$ E staining makes it superior to $10 \%$ NBF. However an elaborate study on the effectiveness of this fixative on special histochemical and immunohistochemical reactions may help in adopting this fixative as a standard histopathological fixative with unaltered characteristics suitable for special techniques and routine surgical pathology laboratory workup.

\section{References}

1. Suresh Durai, Johnsy Merla, Muthuselvi, Shantaraman. Evaluation of a new compound fixative: A step towards limited formalin exposure. Indian journal of pathology and oncology 2017;4(3):413-417.

2. Masir N, Ghoddoosi M, Mansor S, Abdul-Rahman F, Florence CS, Mohamed-Ismail NA, TambyM-R, MdLatar NH. RCL2, a potential formalin substitute for tissue fixation in routine pathological specimens. Histopathology. 2012;60:804-815.

3. Zanini C, Gerbaudo E, Ercole E, Vendramin A, Forni M. Evaluation of two commercial and three home-made fixatives for the substitution of formalin: a formaldehydefree laboratory is possible. Environ Health 2012;11:59.

4. IARC Monographs on the Evaluation of Carcinogenic Risks to Humans, Volume 100F: A Review of Human Carcinogens: Chemical Agents and Related Occupations.

5. Moelans CB, ter Hoeve N, van Ginkel J-W, tenKate FJ, van Diest PJ. Formaldehyde substitute fixatives. Analysis of macroscopy, morphologic analysis, and immunohistochemical analysis. Am J Clin Pathol. 2011;136:548-556.

6. Benerini Gatta L, Cadei M, Balzarini P, Castriciano S, Paroni R, Verzeletti A, Cortellini V, De Ferrari F, Grigolato P. Application of alternative fixatives to formalin in diagnostic pathology. Eur $J$ Histochem.2012;56:e12.

7. Binetti R, Costamagna FM, Marcello I. Development of carcinogenicity classifications and evaluations: the case of formaldehyde. Ann Ist Super Sanita 2006;-42:132-43.

8. Stanta G, Mucelli SP, Petrera F, Bonin S, Bussolati G. A novel fixative improves opportunities of nucleic acids and proteomic analysis in human archive's tissues. Diagn $\mathrm{Mol}$ Pathol 2006;15:115-23.

9. Delfour C, Roger P, Bret C, Berhe ML, Rochaix P, Kalfa N, Raynaud P, Bibeau F, Maudelonde T, Boulle N. RCL 2 , new fixative, preserves morphology and nucleic acid integrity in paraffin embedded breast carcinoma and micro dissected breast tumor cells. J Mol Diagn. 2006;8:157-169.

10. Costa S, Coelho P, Costa C, Silva S, Mayan O, Santos LS, et al. Genotoxic damage in pathology anatomy laboratory workers exposed to formaldehyde. Toxicol 2008;252:40-8.

11. Wiedorn KH, Olert J, Stacy RA, Goldmann T, Kuhl H, Matthus J, Vollmer E, Bosse A. HOPE - a new fixing technique enables preservation and extraction of high molecular weight DNA and RNA of $>20 \mathrm{~kb}$ from paraffin-embedded tissues. Hepes-Glutamic acid buffer mediated Organic solvent Protection effect. Pathol Res Pract 2002;198:735-740.

How to cite this article: Muthuselvi, S, Durai JS, Merla J, Shantaraman K. Evaluation and comparative analysis of minimal formalin containing compound fixatives. Ind $\mathrm{J}$ Pathol Oncol, 2018;5(3):470-476. 